UNDERSTANDING THE ANALOG PUE SIGNALS

BOOSTER TECHNICAL NOTE

NO. 214

D. CIARDULLO

November 30, 1992

ALTERNATING GRADIENT SYNCHROTRON DEPARTMENT BROOKHAVEN NATIONAL LABORATORY UPTON, NEW YORK 11973 


\section{UNDERSTANDING THE ANALOG PUE SIGNALS}

\section{INTRODUCTION:}

Each station of the Booster Beam Position Monitoring (BPM) system has the capability to provide a buffered pair of analog PUE signals (either SUM, DIFF or Plate A, Plate B) to outside users. Although this capability was originally included for specific use by the Damper, Tune Meter and LLRF Radial Control Loop subsystems, the monitoring of analog PUE signals at various locations in the ring has become an increasingly favorite pasttime of machine physicists and operators. As such, the purpose of this technical note is to attempt to convey a better understanding of the abilities and [more importantly] the limitations of the analog PUE link.

A block diagram of the analog link is presented first, followed by a brief discussion of the limitations of each of the main elements comprising the link. These components include the position detector, the BPM system Front End Processing (FEP) electronics, the analog fiberoptic link and the coaxial cable network which delivers the signals to the MCR.

\section{ANALOG CHANNEL DESCRIPTION:}

Figure 1 is a block diagram of the Booster analog link. At each BPM station in the ring, bunched beam passing within the confines of the position detector electrostatically couples onto a pair of Pick-Up Electrodes (PUEs; sometimes loosely referred to as "plates"). The resulting bunch signals are transferred to the Front End Processing (FEP) module of the BPM electronics, where they are scaled and bufferred. The standard PUE hook-ups adopted by the Booster BPM system are as follows:

\section{PUE A: $\quad$ OUTSIDE / TOP \\ PUE B: $\quad$ INSIDE / BOTTOM}

The AUX outputs of the FEP module are then fed to a wideband fiberoptic link, which provides a high degree of coherent noise immunity and isolation from users outside of the BPM system. The bunch signals (or their sum and difference) are again scaled within the fiberoptic transmitter module, this time to match their amplitudes to the dynamic range of the transmitter/receiver pair. The receiving end of the fiber is located in building 914 , room $E$ (upstairs). The output of the fiberoptic receiver is buffered to $50 \Omega$ for transmission to MCR Console 4 via coaxial cable.

\section{THE ANALOG SIGNAL SOURCE:}

The analog signal source at each BPM location in the ring (or BTA line) is comprised of a position detector and a Front End Processing (FEP) module. The relationship between the charge contained in a passing bunch and the output of the FEP module can be found in the reference ${ }^{1,2}$. When using the auxilliary FEP outputs, the transverse position of the bunch can be approximated by 


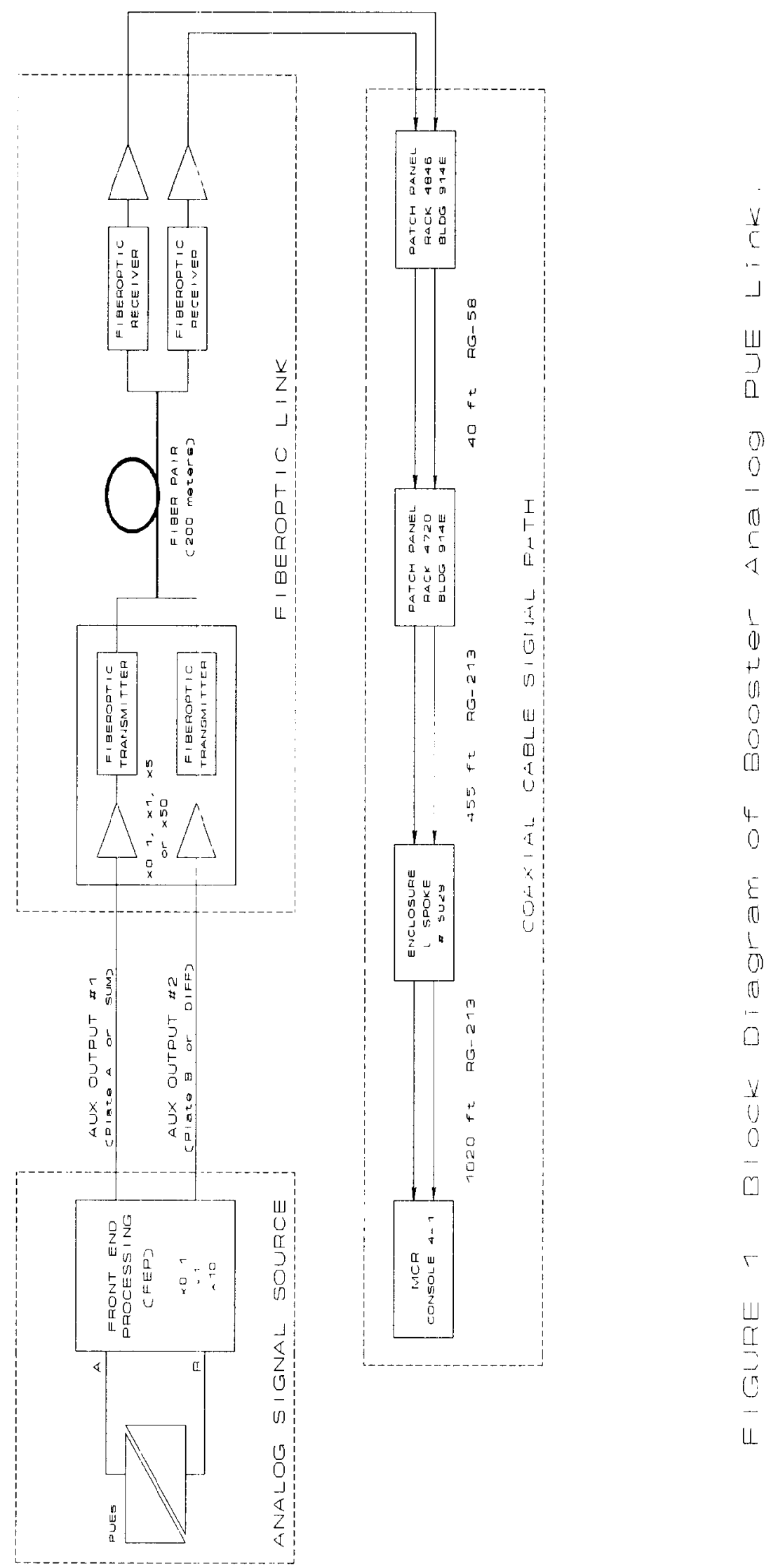




$$
\text { Position }=K \frac{\left(V_{A}-V_{B}\right)}{\left(V_{A}+V_{B}\right)}=K \frac{V_{\Delta}}{V_{\Sigma}}
$$

where $V_{A}$ and $V_{B}$ are the peak PUE bunch signal voltages

$V_{\Sigma}$ is the peak AUX SUM signal voltage, and

$\mathrm{V}_{\triangle}$ is the peak AUX DIFF signal voltage.

In the above relation, $\mathrm{K}$ is a constant which includes the differential sensitivity of the detector (which is FEP gain mode dependent), as well as a gain correction factor for its associated FEP module. Both the detector and the front end electronics will be discussed separately, and the impact each has in determining the value for $\mathrm{K}$ in Eq.[1] will be examined.

\section{The Position Detector}

All BPM locations in the Booster ring and BTA line utilize electrostatic split cylinder PUEs. Although each detector has a verified linearity of $\pm 0.1 \mathrm{~mm}$ within an aperture of $\pm 30 \mathrm{~mm}^{3,4}$, the typical detector sustains this performance to $\pm 50 \mathrm{~mm}$. In addition, the detectors have been designed to maintain their accuracy after repeated $200^{\circ} \mathrm{C}$ bakeouts. The design of the detector includes a calibration ring which couples equally to both electrodes, providing a useful test port which can be used to align the entire analog channel. Note, however, that the calibration ring simulates only one particular beam position....centered. Although the cal ring may be used to remotely verify connection of the FEP module to the detector, it is not possible to distinguish which channel of the processing electronics is connected to which PUE (a subtle but important point).

Since the detector used in the Booster is the electrostatic type, the signal voltage induced on a given set of PUEs depends upon the capacitive loading. This net capacitive load is presented to the plates by the complex input impedance of the front end electronics and its associated connecting cables. Both channels (one channel per PUE) of a given FEP module are matched to effect an equal impedance to the PUE pair of the detector; However, the absolute impedances from module to module (i.e., at different locations within the ring) are not matched. Thus the value obtained from the outputs of differing FEP modules may vary by a few percent. Using DIFF/SUM normalization would at first appear to remove any effect this has on the calculation of beam position, since the same electrode voltages are used to determine both the sum and the difference signals. Unfortunately, reactive loading of the PUEs does in fact affect the differential sensitivity of the detector, and hence the position calculation. The relation describing this effect is

$$
K=K_{0}\left[1+\frac{2 C_{p p}}{C_{L}+\left(C_{p}+C_{p C}\right)}\right]
$$

where $\mathrm{K}$ is the effective (inverse) differential sensitivity of the reactively loaded detector

$\mathrm{K}_{0}$ is the initial (inverse) diff sensitivity of the detector, based on its geometry [89.7 $\mathrm{mm}$ ]

$\mathrm{C}_{\mathrm{L}}$ is the capacitive load on the detector PUE [275pF, 2675pF]

$\mathrm{C}_{\mathrm{p}}$ is the capacity from one PUE to the detector shell [26pF]

$\mathrm{C}_{\mathrm{pc}}$ is the capacity of the PUE to the calibration ring [10pF] and

$\mathrm{C}_{\mathrm{pp}}$ is the plate to plate capacitance [18pF] 
This relation has been verified experimentally, during the period of detector testing prior to Booster commissioning. The number shown in brackets represents the typical approximate value for each of the relevant capacitances found in the detector model ${ }^{5}$. In the case of $\mathrm{C}_{\mathrm{L}}$, the loading depends on whether the FEP module is in $\mathrm{x} 0.1$ mode [nominal $2675 \mathrm{pF}$ ], $\mathrm{x} 1$ mode [nominal $275 \mathrm{pF}$ ] or $\times 10$ mode [nominal $275 \mathrm{pF}$ ]. Figure 2 is a plot of Eq.[2], utilizing the detector capacitance constants listed above. Note that for "high intensity" measurements (i.e., orbits taken with the FEP module in x0.1 mode) the nominal reactive load presented to the PUEs is $2,675 \mathrm{pF}$, which is located on the flatter region of the graph. This indicates a value of detector $\mathrm{K}$ which is relatively insensitive to capacitance load variations. Since the differential sensitivity remains fairly constant from detector to detector, the result is a more consistent position measurement at all locations. In the $\mathrm{x} 1$ and $\mathrm{x} 10$ modes, however, the smaller capacitance load of $275 \mathrm{pF}$ puts the operating point on a steeper portion of the curve. This results in values of $\mathrm{K}$ with a higher sensitivity to capacitance load variations. Since the front end modules are not matched from unit to unit, some variation can be expected in the value of $\mathrm{K}$ from one detector to the next while in one of these gain modes. Since position is linear with K (Eq.[1]), the position error due to this effect increases as the beam moves transversely away from beampipe center.

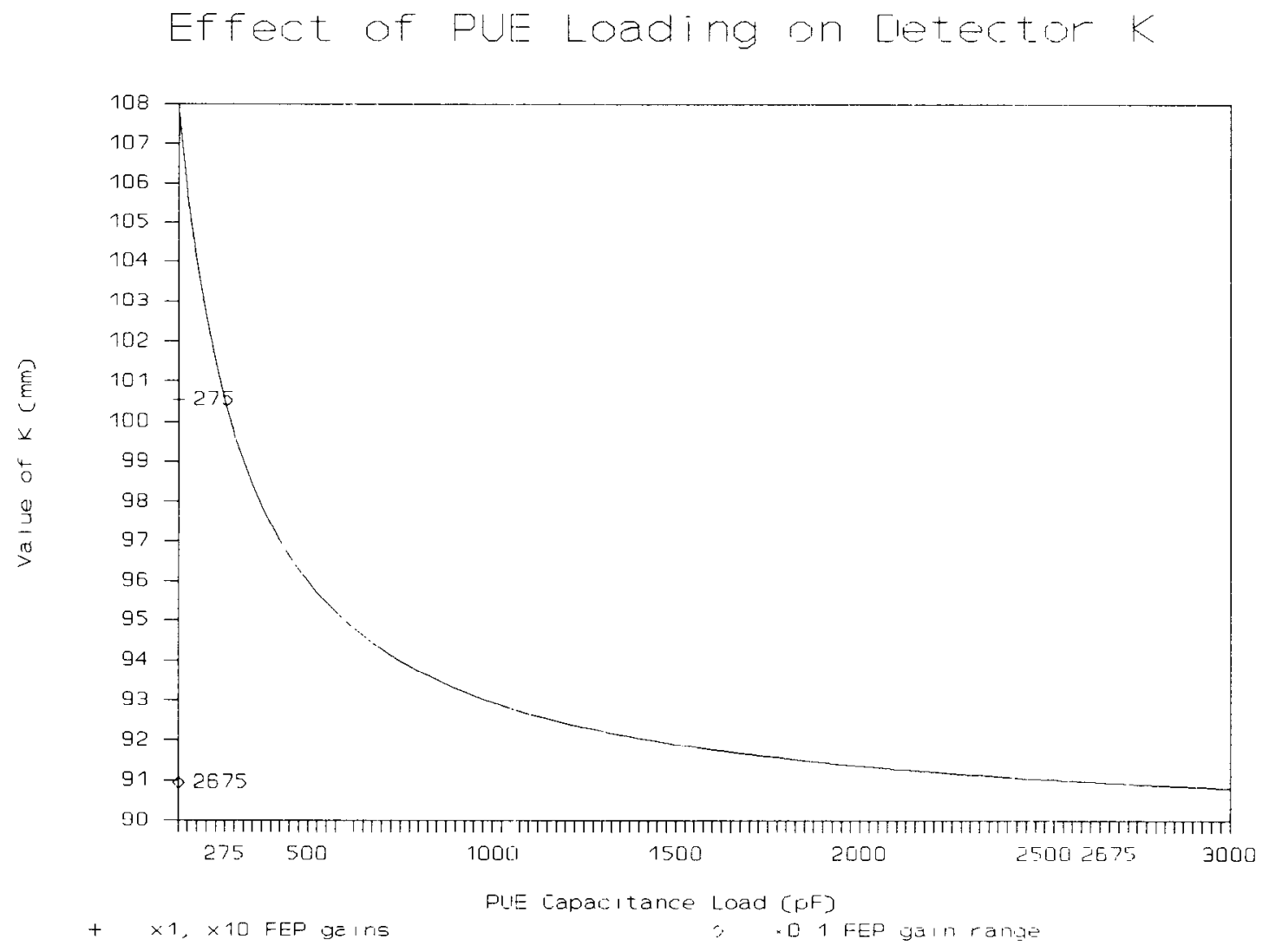

Figure 2. PUE Capacitance Loading Effect on Detector K

\section{The Front End Processing (FEP) module}

The FEP module is the interface between the PUEs and the data acquisition electronics ${ }^{6}$, and has several functions. The module preserves the temporal response of the bunch signals by appropriately matching the electrode impedance to that of the electronics. A flat amplitude response with frequency 
results, and the bunch signals maintain their original "time domain" shape factor. The FEP has three gain settings controllable via spreadsheet $(\mathrm{x} 10, \mathrm{x} 1$ and $\mathrm{x} 0.1)$, and provides real-time sum and difference processing. Each module has a set of auxilliary outputs which can be configured to provide either the bunch signals or their sum and difference. The dynamic range within any one gain setting is approximately $40 \mathrm{~dB}$ for the Plate A, Plate B and SUM outputs. The frequency response of the FEP electronics when using the AUX SUM and DIFF outputs is from $50 \mathrm{kHz}$ to $20 \mathrm{MHz}$. The AUX Plate A and Plate B outputs have a $-3 \mathrm{~dB}$ frequency response of approximately $12 \mathrm{kHz}$ to $70 \mathrm{MHz}$ for the $\mathrm{x} 1$ and $\mathrm{x} 10$ gain modes, and $1.2 \mathrm{kHz}$ to $70 \mathrm{MHz}$ in $\mathrm{x} 0.1$ mode. The module can detect beam intensities as low as $10^{9} \mathrm{ppp}$ (providing the signal's spectral content falls within the frequency response of the $\mathrm{x} 10$ gain mode).

The absolute accuracy of the front end electronics is a function of its common mode performance (which affects where the electronics "thinks" the beampipe center is), as well as the reactive load that the module presents to the PUEs. The common mode performance determines the accuracy of the electrical center of the PUEs; In the case of the Booster front ends, the common mode performance out of the auxilliary outputs is typically better than $-45 \mathrm{~dB}$, corresponding to a fraction of a millimeter position offset. [Better performance is available from the BPM system outputs, used in the orbit monitoring program]. The design philosophy was to match both input channels of a given FEP module such that common-mode performance was maximized over all three gain ranges. No attempt was made to match the input impedance from one module to another. Consequently, there will be small variations in the effective detector differential sensitivity used to calculate the beam position (see preceeding section describing the position detector).

When utilizing the AUX SUM and AUX DIFF outputs (as opposed to the Plate A and Plate B bunch signal outputs) there is extra gain included in the difference channel which needs to be accounted for in the position calculation. The difference channel has (on average) a factor of $x 1.914$ more gain than the SUM channel, including transformer losses. The value of $\mathrm{K}$ used in the position calculation of Eq.[1] must be divided by 1.94 to correct for the gain imbalance of the two channels. This imbalance is a function of the sum/diff hybrid, and hence only needs to be accounted for when using the AUX SUM and DIFF outputs.

\section{THE FIBEROPTIC LINK:}

The "fiberoptic link" used in the Booster consists of a Meret Inc. MDL288TV analog f/o transmitter/receiver pair with 200 meters of Ensign-Bickford HCR series fiber connecting them. (Details about the link can be found in the reference ${ }^{7}$ ). Also included as part of the $\mathrm{f} / \mathrm{o}$ link are an adjustable gain pre-amplifier and post amplifier/buffer (both BNL made). The post amp is located in building 914E, and serves as a buffer to drive the long $50 \Omega$ transmission line back to the MCR. Since the dynamic range of the FEP module is much greater than that of the Meret xmit/rec pair $(40 \mathrm{~dB}$ in any single gain range for the FEP, as opposed to $26 \mathrm{~dB}$ for the $\mathrm{f} / \mathrm{o}$ link), it was found necessary to include a pre-amplifier in front of the transmitter. Adjustable amplifier gain helps to maximize the useful dynamic range of the f/o link by matching it to the expected PUE bunch signal levels from the FEP output. Gains of $x 0.1, \times 1, \times 5$ and x50 are remotely selectable via spreadsheet. Two channels, each consisting of a pre-amp and Meret f/o transmitter, are packaged in one module. This module resides in the local BPM electronics crate at the Booster location of interest. The optical output of both channels are transmitted to the receivers in building $914 \mathrm{E}$ via dual fiber cable.

There is currently no method of remotely "fine-tuning" the gain match of the two channels of a given dual fiberoptic link. Therefore, the user should verify that the gains of both links are sufficiently matched before recording data (it is possible to use built-in test signals from the BPM system to drive the 


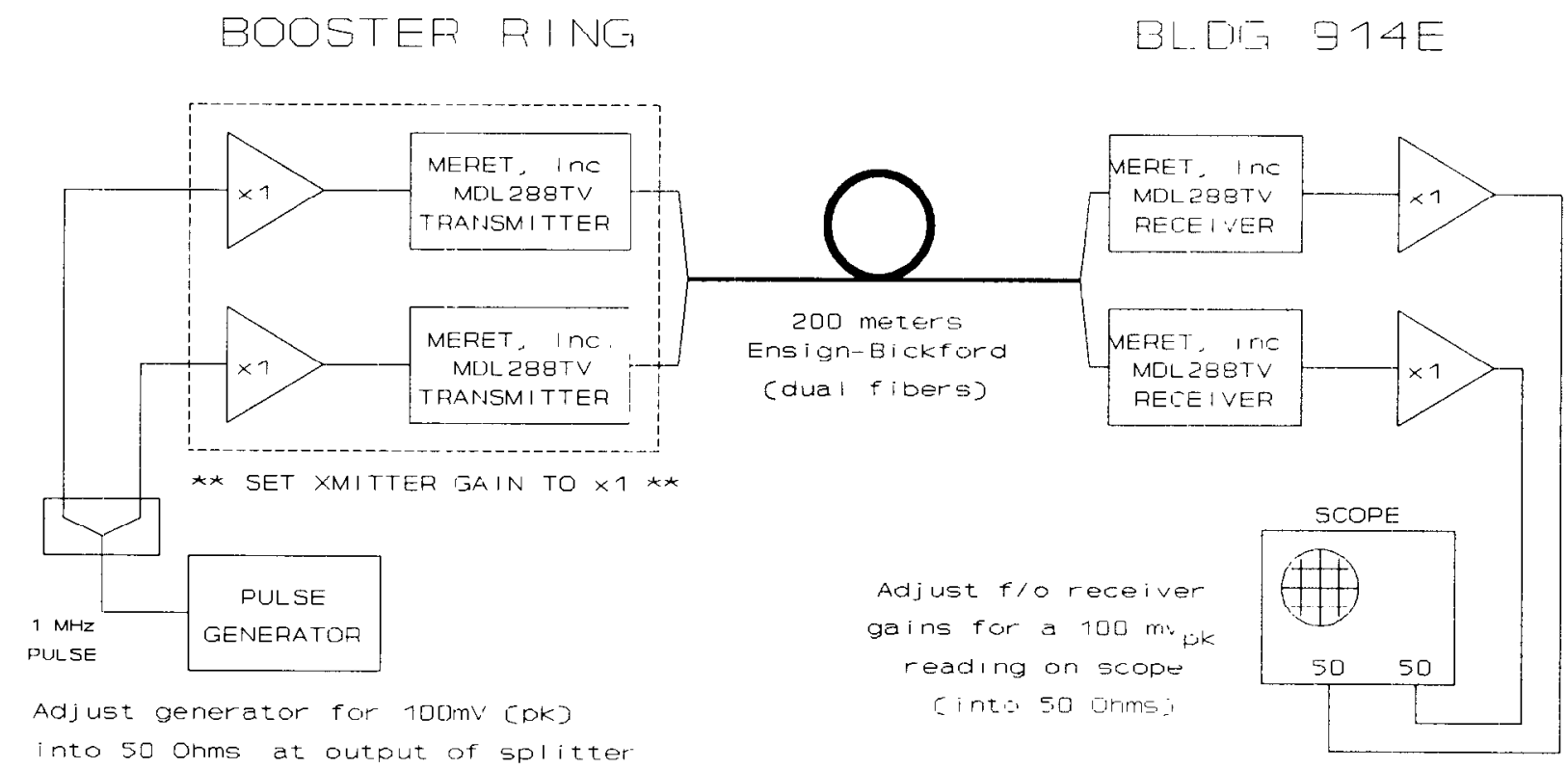

Figure 3. Alignment Set-up for the Analog Fiberoptic Link.

calibration ring for this purpose). Note that two f/o channels which appear matched at one pre-amp gain may not necessarily remain matched if the gain of the pre-amp is changed. In addition, the user is cautioned that signals greater than $0.5 \mathrm{~V}_{\mathrm{pk}}$ input to the Meret $\mathrm{f} / \mathrm{o}$ transmitter will cause excessive distortion to the bunch signal. Note from Figure 1, however, that by the time the signal reaches MCR 4 , its amplitude will already have been modified due to coaxial cable losses as well as gain (or loss) due to the fiberoptic link itself; Therefore, a $0.5 \mathrm{~V}_{\mathrm{pk}}$ signal amplitude at MCR 4 does not, in general, correspond to a $0.5 \mathrm{~V}_{\mathrm{pk}}$ amplitude at the input to the f/o transmitter. Figure 3 illustrates the method which should be used to "set-up" and calibrate a pair of fiberoptic channels prior to use. A signal generator set to output a $1 \mathrm{MHz}$ pulse with amplitude $100 \mathrm{mV}_{\mathrm{pk}}$ (well within the linear range of the Meret link) is input to the fiberoptic transmitter module, located in the ring. With the f/o preamp gain set to $x 1$, the gain of the Meret xmitter/receiver pair is adjusted to obtain an overall link gain (from the pre-amp input in the ring to the buffer output in building 914E) of $x 1$. This adjustment is made at the optical receivers in $914 \mathrm{E}$. With a f/o link gain of $\mathrm{x} 1$ between the Booster and $914 \mathrm{E}$, a more confident estimate of the input level to the Meret transmitters can be made "on the fly".

\section{COAXIAL DELIVERY OF SIGNALS TO MCR:}

Bunch signals (or their sum and difference) are transported to MCR 4 from builing 914E via $50 \Omega$ coaxial cable. Figure 1 shows the path of the signals ${ }^{8}$ (each box in the figure represents a connection point). The bunch signals being carried along these transmission lines typically have a bandwidth on the order of 20MHz; And since the cable run is long (1475 ft. of RG-213 in series with $40 \mathrm{ft}$. of RG-58), significant frequency dependent attenuation will occur. The twelve cable runs have the same length, implying that their frequency response should have little effect on position calculations using the peak signal voltages received at MCR 4. The cable response does, however, hamper efforts to estimate the input amplitude to the Meret fiberoptic transmitters, due to the varying spectral content of the bunch signals throughout the acceleration cycle. (Recall that it is necessary to limit the input signal voltage in order to prevent f/o link saturation). As a rough reference, an $80 \mathrm{nsec}$ square pulse with $4 \mathrm{MHz}$ prf was measured to have approximately $5 \mathrm{~dB}$ of attenuation (as measured to the waveform peak), with significant 
temporal distortion. If this signal were being supplied by the analog fiberoptic link (set to a link gain of $\mathrm{x} 1$ as outlined in Fig.3), a $500 \mathrm{mV}_{\mathrm{pk}}$ signal at the input to the Meret transmitter would show up as a 284 $\mathrm{mV}_{\mathrm{pk}}$ distorted version of the waveform at MCR 4. Therefore, as a loose "rule of thumb", users of the analog PUE link are advised to limit the amplitude of signals received at MCR 4 to voltages below 250 $\mathrm{mV}_{\mathrm{pp}}$. (The restriction is quoted as $\mathrm{V}_{\mathrm{pp}}$ because the entire analog channel is AC coupled). This can be accomplished by changing the gain of the fiberoptic preamplifiers and/or FEP gain as necessary.

\section{SUMMARY:}

\section{Detector/FEP Combination}

1. Common-mode performance of the FEP determines the accuracy of the electrical center of the PUEs (where the electronics thinks the center is); $-45 \mathrm{~dB}$ is a typical value; corresponding to an accuracy of approximately $0.56 \mathrm{~mm}$.

2. Variations in the capacitance loading of a given set of PUEs affects the apparent differential sensitivity of that detector. This implies that there will one value of $\mathrm{K}$ in Eq. [1] for the $\mathrm{x} 1$ and $x 10$ FEP gain modes, and another for the $x 0.1$ mode. Equivalent input capacitance measurements have been made on the majority of the installed FEP modules; Using Eq.[2] (the effective value of $\mathrm{K}$ due to PUE loading), these measurements translate to the following expected values of $\mathrm{K}$ :

$\begin{array}{ll}\frac{\text { FEP Gain Mode }}{\mathrm{x} 1, \mathrm{x} 10} & \frac{\text { Range of } \mathrm{K}}{100.9 \mathrm{~mm}-99.34 \mathrm{~mm}} \\ \mathrm{x} 0.1 & 90.97 \mathrm{~mm}-90.89 \mathrm{~mm}\end{array}$

The error due to this effect is seen to be negligible for the high intensity (x0.1) gain mode, but can result in position descrepancies of up to $0.5 \mathrm{~mm}$ from detector to detector when the FEP is in the $\mathrm{x} 1$ or $\mathrm{x} 10$ gain modes. This error is position dependent; i.e., it is negligible at the center position, and increases linearly as the beam moves off center toward the aperture.

3. When using the AUX SUM and DIFF outputs of the FEP, the value of $\mathrm{K}$ used in the position calculation of Eq.[1] must be divided by 1.914 to correct for the gain imbalance between the DIFF and SUM channels. This imbalance is a function of the sum/diff hybrid, and does not need to be accounted for when utilizing the bunch signal (Plate A and Plate B) outputs.

\section{Fiberoptic Link}

1. The peak bunch signal voltage into the Meret $\mathrm{f} / \mathrm{o}$ transmitter should never exceed $0.5 \mathrm{~V}_{\mathrm{pk}}$, as this will cause sufficient signal distortion to affect position measurements made at MCR 4 . The recommended "rule of thumb" is to maintain a peak bunch signal amplitude of $\leq 250 \mathrm{mV}_{\mathrm{pp}}$ at MCR 4. This can be accomplished by manipulating the gain of the f/o preamp and/or the FEP electronics.

2. The f/o link from the preamp (in the ring) to the output of the buffers (in 914E) should be adjusted for an overall gain of $x 1$. Using the calibration procedure illustrated in Figure 3 serves to match the gain of both channels, and allows the user to make a more reliable prediction regarding the signal level entering the Meret transmitter. Link calibration can be verified remotely using the built-in calibration pulses of the BPM system. A calibration should always be performed prior to taking data at MCR Console 4, especially if it is suspected that the fiber connection to 
either the Meret transmitter or receiver has been disturbed (connector alignment is critical, and can affect overall signal gain through the optical link).

\section{REFERENCES:}

1. D.J. Ciardullo, "An Estimate of the Signals Appearing at the Input to the AGS Booster Beam Position Monitoring System," BNL Booster Technical Note \#170, July 11, 1990.

2. D. Ciardullo, "Estimating Beam Intensity from the Booster BPM System," Booster Technical Note \#206, February 11, 1992.

3. R. Thomas, D.J. Ciardullo, W. Van Zwienen; "Design and Testing of the AGS Booster BPM Detector," in Proceedings of the 1991 IEEE Particle Accelerator Conference, Vol. 3, Chicago, IL, March 1989, pp.1344-1346.

4. D.J. Ciardullo, R.A. Thomas; "Pre-installation Scan Measurements of the AGS Booster Electrostatic Beam Position Monitors," BNL Booster Technical Note \#201, October 21, 1991.

5. D.J. Ciardullo, "Low Frequency Capacitance Measurements of the AGS Booster Electrostatic Pick-Up Electrodes," BNL Booster Technical Note \#178, September 13, 1990.

6. D.J. Ciardullo, G.A. Smith, E.R. Beadle, "Design of the AGS Booster Beam Position Monitor Electronics," in Proceedings of the 1991 IEEE Particle Accelerator Conference, Vol. 3, Chicago, IL, March 1989, pp.1431-1433.

7. E.R. Beadle, "Fiber Optics in the BNL Booster Radiation Environment," in Proceedings of the 1991 IEEE Particle Accelerator Conference, Vol. 3, Chicago, IL, March 1989, pp.1493-1495.

8. R. DiFranco, private communication. 